\title{
Structure of Electrorheological Fluids
}

\author{
U.Dassanayake and S.Fraden \\ Complex Fluids Group, Martin Fisher School of Physics, Brandeis University, Waltham, MA \\ 02454 \\ A. van Blaaderen \\ Van 't Hoff Laboratory, Debye Inst., Utrecht University, The Netherlands and FOM Inst. for \\ Atomic and Molecular Physics, Amsterdam, The Netherlands
}

(October 23, 2018)

\begin{abstract}
Specially synthesized silica colloidal spheres with fluorescent cores were used as model electrorheological fluids to experimentally explore structure formation and evolution under conditions of no shear. Using Confocal Scanning Laser Microscopy we measured the location of each colloid in three dimensions. We observed an equilibrium body-centered tetragonal phase and several non-equilibrium structures such as sheet-like labyrinths and isolated chains of colloids. The formation of non-equilibrium structures was studied as a function of the volume fraction, electric field strength and starting configuration of the colloid. We compare our observations to previous experiments, simulations and calculations.
\end{abstract}




\section{INTRODUCTION}

Electrorheological (ER) fluids are suspensions of dielectric particles (usually of size 1$100 \mu \mathrm{m})$ in non-conducting or weakly conducting solvents. For particles with radii below several $\mu \mathrm{m}$ Brownian motion is still important and such dispersions are called colloidal. When electric fields are applied across these suspensions they tend to show altered viscous behaviour above a critical value of the electric field, with the apparent viscosities increasing by several orders of magnitude at low shear rates. Above this critical electric field, at low shear stresses the suspensions behave like solids, and at stresses greater than a 'yield stress' the suspensions flow with enhanced viscosity. The rheological response is observed to occur in milliseconds, and is reversible. This combination of electrical and rheological properties has led to many proposals for applications of ER fluids in such devices as hydraulic valves, clutches, brakes, and recently in photonic devices [1] 3.

Application of an electric field results in structural transitions in the colloidal suspension because the interparticle electrostatic interactions due to polarization are stronger than Brownian forces. The tendency of particles in suspension to form structures such as chains upon application of an electric field was reported centuries ago by scientists such as Franklin and Priestly [6. [5]. Quantitative experiments on the electrorheological effect were first performed by Winslow in 1949, when he reported that suspensions of silica gel particles in low-viscosity oils tend to fibrillate upon application of electric fields, with fibers forming parallel to the field [6]. Winslow reported that at fields larger than $\approx 3 \mathrm{kV} / \mathrm{mm}$ the suspensions behaved like a solid, which flowed like a viscous fluid above a yield stress that was proportional to the square of the applied electric field.

Particle association is the main cause of the altered rheological behaviour of ER fluids. The nature of the field induced structures are an important factor in determining the yield stress and flow behaviour. Moreover, theoretical studies of the rheological properties of ER fluids are typically performed by subjecting possible field induced structures to shear stresses and obtaining stress-strain relationships. Therefore it is important to experimentally study the nature of the particle aggregates quantitatively in real-space. In this paper we describe 
an experimental study of structure formation in a model ER fluid in the absence of shear fields, by direct visualization of the fluid.

A recent and comprehensive survey of ER fluids, where the issue of particle aggregation is addressed, is provided by Parthasarathy and Klingenberg [7]. Tao and Sun have predicted the (zero-temperature) ground state for ER fluids to be a body centered tetragonal (BCT) structure (see figure 1). Their result was obtained for suspensions of uniform spheres by treating the ER fluid as a suspension of point dipoles in a dielectric fluid and by only taking energy considerations (neglecting entropy considerations) into account [8]. An experimental verification of this proposed ground state structure has been provided by laser diffraction studies of ER systems consisting of $20 \mu \mathrm{m}$ diameter glass spheres suspended in silicone oil, where entropy considerations are indeed not important [9]. Halsey and Toor [10] have described the evolution of structure as occurring in two identifiable stages. The particles first chain along the electric field, and then aggregate into dense structures that take the form of columns aligned with the electric field. In recent computer simulation studies, Martin, Anderson and Tigges have observed the formation of two-dimensional 'sheets' of particles (described later) as an intermediate state in structure evolution [11,12]. In their simulations, Martin and coworkers describe the evolution of structure in an ER fluid consisting of 10,000 particles over a concentration range of $10-50 \%$ volume fraction. They study the mechanics of coarsening and the emergence of crystallinity, and use various methods of characterizing the structures that evolve, including pair correlation functions, microcrystallinity and coordination number. Several aspects of their simulation results are evocative of our observations, but there are several notable exceptions as described below.

\section{METHODS}

The model ER fluid used in our experiments was a solution of monodisperse silica spheres $(0.525 \mu \mathrm{m}$ radius, polydispersity in size $1.8 \%)$ in a mixture of water and glycerol. The spheres were charged and this prevented irreversible aggregation. The spheres had fluorescent cores of $0.4 \mu \mathrm{m}$ diameter and were labeled by fluorescein isothiocyanate (FITC). The colloids 
were the same as used in previous work in which particle coordinates were obtained in bulk samples [13,14. The non-fluorescent layer surrounding the core made it possible to distinguish individual particles even when they were in contact with each other. The synthesis of this kind of spheres is described in ref. [15]. Interparticle attraction was reduced by matching the refractive index of the solvent to that of the particles, minimizing van der Waals forces. The matching of refractive indices also enabled visualization in the bulk of concentrated samples by reducing the multiple scattering of light. The silica spheres had a refractive index of 1.45, and index matching was achieved by using a solvent mixture of 16 w.\% water (refractive index 1.33) and 84 w.\% glycerol (refractive index 1.48). The viscosity of such a mixture $(84.4 \mathrm{cP})$ also allows a larger time window in which the dynamics of structure formation can be followed with confocal microscopy. We estimate that the silica spheres had a dielectric constant of 3.7 (at $500 \mathrm{kHz})$ while the solvent had a dielectric constant of $\approx 49($ at $500 \mathrm{kHz})$, values which are close to the values at $0 \mathrm{~Hz}$. While the refractive indices were approximately matched at light frequencies, we could still obtain a dielectric mismatch between the spheres and the solvent at frequencies in the $500 \mathrm{kHz}$ range in which we applied AC electric fields. The ER fluid was placed in a cell, which consisted of parallel microscope slides that were coated with indium tin oxide (ITO), a transparent conductor. The ITO coating had an electrical resistance of $100 \Omega$ per square inch. One slide was $\approx 1.2 \mathrm{~mm}$ thick, while the other was $\approx 150 \mu \mathrm{m}$ thick, and served as a cover slip. An insulating sheet of Kapton (DuPont) was placed between the electrodes, serving as a means for adjusting the electrode gap (between $5-100 \mu \mathrm{m}$ ). The ER fluid was placed in a $\approx 3 \mathrm{~mm}$ diameter hole cut out of the Kapton sheet, which served to confine the fluid, and the electrodes were aligned horizontally.

The electrodes were connected to a power supply providing a uniform electric field. Electric fields of strength $\approx 1 \mathrm{kV} / \mathrm{mm}$ and frequency $\approx 500 \mathrm{kHz}$ were used. A Krohn-Hite (Avon, MA) Model 7602M wideband power amplifier and a waveform generator from Wavetek (San Diego, CA) were used to produce the electric fields. The electric field strength was of the same order of magnitude as that used in previous experiments on ER fluids. The frequency was chosen so that effects due to the polarization of the double layer could be neglected, 
and the polarization of the spheres could be attributed to the dielectric mismatch between the spheres and the solvent.

The observations were made using confocal scanning laser microscopy (CSLM) [16] which is a technique that images individual planes in a sample by rejecting most of the fluorescent light emitted by particles out of the imaging plane. Additionally, confocal microscopy yields better resolution than conventional light microscopy both along the optical axis and in the image plane. The resolution produced is $\approx 0.6 \mu \mathrm{m}$ along the optical axis, and $\approx 0.2 \mu \mathrm{m}$ in the image plane. In our experiments we used CSLM to obtain sequences of digitized 2 -dimensional images of the sample at planes separated by $\approx 0.1 \mu \mathrm{m}$. These images were then computer-analyzed to obtain the 3-dimensional coordinates of the particles present in the sample. These coordinates could then be used to analyze the structure, and to render the sample as a 3-dimensional graphical object. Typically it took several minutes to obtain a three dimensional data set of $512 \times 512 \times 100$ voxels (e.g., $40 \mu \mathrm{m} \times 40 \mu \mathrm{m} \times 20 \mu \mathrm{m}$ ) and several seconds to obtain a single 2D image plane of $1024 \mathrm{x} 1024$ pixels (e.g., $40 \mu \mathrm{m} \times 40 \mu \mathrm{m}$ ) either as a plane perpendicular or parallel to the optic axis. The experiments were performed on a system consisting of a Leica inverted microscope with a 100x1.4NA oil lens with a Leica TCS confocal attachment.

The process of obtaining the 3-dimensional coordinates of the particles from the confocal microscope images was similar to that described in [13,14], and is illustrated schematically in figure 2. The stacks of images were of sequential planes perpendicular to the optical axis, about $0.1 \mu \mathrm{m}$ apart. The (spherical) particles imaged through the confocal system appear as circular regions in the digitized images (see figures $3,4,5$ ) because of the circular symmetry of the confocal microscope point spread function(psf) in the plane perpendicular to the optical axis (the xy plane). Because of the finite extent of the psf along the optical axis ( $\mathrm{z}$ axis), a given particle is imaged in several consecutive image planes as circular regions of varying intensity and size. Each image was first analyzed to find the centers of each region present. This was achieved by identifying each region above a chosen threshold of intensity as resulting from an individual particle, and identifying the intensity-weighted average position 
(xy coordinate) of each region as the center (care was taken to identify overlapping particle regions). Neighbouring planes were then looked at, and region centers with approximately the same xy coordinates were identified as belonging to a single particle, forming a 'string' of centers for a single particle. The final xyz coordinates of each particle was obtained by finding the center of intensity along the 'strings'. Since there is a distribution in the sizes of the cores there is also a distribution in the fluorescent intensities of the particles. Furthermore, the fluorescence photobleaches, and the detected intensities diminish in the bulk of the sample due to optical abberations. Therefore the algorithm had to be applied at many iterations of the threshold value. The initial threshold value was set at the intensity of the most weakly fluorescing particles. These were then identified as distinct particles using the above described algorithm, while particles of higher intensity blended together. Once the weakest particles were identified, they were removed from the dataset, the threshold was increased, and the search for particles was repeated. This was repeated at several iterations of the threshold value. The particle coordinates found were verified by marking the calculated coordinates in the raw data and visually inspecting the results. The data was rendered in

3 dimensions on a Silicon Graphics Indigo ${ }^{\mathrm{TM}}$ platform using programs implementing native graphics library routines. Data was also visualized through web-based $3 \mathrm{~d}$ browsers using the VRML format (specified at http://www.vrml.org). IDL, a programming environment useful for visual data analysis (from Research Systems Inc., Boulder, CO), was used extensively for the manipulation of images.

\section{OBSERVATIONS}

We found the structure formation to proceed through a sequence of nonequilibrium structures depending on the initial conditions of the suspension and the strength of the applied field. The structure formation occurred most rapidly at early times, within a few seconds. We found it most useful to describe the structure development according to the volume fraction of spheres used. The following is a summary of our observations.

At low fields, below $\approx 100 \mathrm{~V} / \mathrm{mm}$, where the interparticle electrostatic interaction energies 
were low compared to thermal energies, no significant particle association was observed. The spheres tended to sediment to the bottom electrode, having a density larger than the glycerolwater solvent. The structural observations were performed at field strengths of $\approx 1000 \mathrm{~V} / \mathrm{mm}$ where field induced structures, such as chains of touching particles, that formed were not observed to break up due to thermal fluctuations, implying that the electrostatic energy at contact was many kT. However, we observed significant Brownian motion even in the final crystalline states. A particle in a BCT crystal with an applied field of $0.5 \mathrm{kV} / \mathrm{mm}$ typically had a measured mean square displacement (in the plane perpendicular to the electric field) of about $5 \%$ of the lattice spacing.

At the lowest observed particle volume fractions of about $10 \%$, and with the particles initially distributed throughout the volume of the solvent, rapid application of fields of strength $\approx 1000 \mathrm{~V} / \mathrm{mm}$ resulted in the formation of field-aligned chains and 'sheets'. Chains are linear associates of touching spheres aligned along the field, with a wide variation in length. Sheets are hexagonally ordered, 2-dimensional structures, aligned with the field direction (figure 3). At this particle concentration the number of chains formed initially was larger than the number of sheets, and as the concentration was increased, the presence of chains decreased relative to the presence of sheets. Sheets appeared within seconds of the field being turned on, and rapidly formed into a complex, interconnected labyrinthine formation (figure 4a). They formed initially at the electrodes and grew away from both electrodes towards the middle of the electrode gap. The chains appeared initially throughout the sample, with more in the middle of the electrode gap where they rapidly transformed into BCT structures by attracting each other. We determined the structures to be BCT by direct measurement of the crystal dimensions (figures 5,6). The sheets transformed into BCT structures over a time of a few hours by annealing together, beginning in the regions away from the electrodes, and growing towards the electrodes. The BCT structures that formed rearranged themselves into a network of misaligned BCT regions such as seen in figure $4 \mathrm{~b}$ and only coarsened very slowly over the time of observation of 1-2 days by collective motions. The first 2-3 layers of spheres at the electrodes remained in hexagonal planes parallel to the 
electrodes throughout the observation (figure 6). An explanation may be that the spheres at the electrodes are strongly attracted to their image dipoles created by the conducting electrodes. We did not have enough data of particle coordinates to attempt to characterize the structure in terms of a local order parameter and thereby to quantify the structure formation over time [1]], although in principle this is possible to do.

Table 1 shows calculated values of the dipolar energy per particle at large electrode gaps for various particle arrangements (from refs [8] and [11]). The energy per particle is in units of $p^{2} / a^{3} \epsilon_{f}$, where $p=a^{3} \epsilon_{f} \mathrm{E}\left(\epsilon_{p}-\epsilon_{f}\right) /\left(\epsilon_{p}+2 \epsilon_{f}\right) . \epsilon_{p}$ and $\epsilon_{f}$ are the dielectric constants of the particle and solvent respectively, and $\mathrm{E}$ is the magnitude of the electric field. The BCT structure is the most favourable structure, while sheets have a value in between that of chains and BCT. The value given for sheets is that for large hexagonally packed sheets and is independent of the arrangement of the spheres within the sheets. Neglecting the presence of the electrodes, because of the symmetry, the dipolar energy per particle in a hexagonally packed planar field-aligned sheet is independent of its orientation [17]. However, in our observations we saw a distinctive orientation for the sheets. As seen in figure 3, the sheets we observed can be considered to be composed of strings of nearest neighbours of spheres that are tilted by $30^{\circ}$ with respect to the electric field axis (as opposed to our expectations of observing hexagonal packing created by a series of offset sphere chains aligned with the field). This observed orientation for the sheets is probably created by the presence of the electrodes. Because of the strong attraction between spheres and their image dipoles at the electrodes, a layer of spheres forms on the electrode, which nucleates the growth of hexagonal sheets having the configuration observed. Figure 7 shows a calculation of the energy per dipole (in units of $p^{2} / a^{3} \epsilon_{f}$ ) for various structures where the calculations include the presence of the electrodes by including interactions with image dipoles. The calculations assume fixed identical dipoles interacting with the external field and each other. For each structure considered, the value given is that for a dipole located in the center of the structure. Two configurations of sheets are considered, the observed configuration and that formed by a close packed arrangement of chains. For electrode gaps smaller than $\approx 10$ sphere diameters 
the observed sheet structure shows the lowest energy. At larger electrode gaps the BCT structure has the lowest energy, while the difference between the values for the two sheet configurations decreases. Our experiments agree with this simple calculation which neglects entropy contributions. For electrode gaps less than $\approx 12$ sphere diameters we observed that the sheets (and a smaller population of chains) that formed upon application of the field persisted over the duration of observation (2 days) and were the dominant structure present, with a small fraction of spheres forming into BCT crystals. Thus experimentally the sheets seemed to be the equilibrium structures at these electrode gaps. It is interesting to note that while it would seem natural for chains of particles to aggregate together to form hexagonal sheets, we did not observe this in our experiments. Thus the small energy gain (due to the presence of the electrodes) in forming our observed sheets, while decreasing with increasing electrode gap, was most likely sufficient to select a preferred orientation for the sheets, even in very thick samples.

As the particle volume fractions in our samples were increased from $10 \%$ to $15 \%$, the presence of chains decreased, and increasing proportions of the sample adopted the metastable sheet state. The time for the sheets to transform into BCT structures increased as the applied E-field value was increased. We did not have enough time resolution to observe the mechanics of the formation of sheets, which formed within seconds. When the E-field was turned off, the structures disassociated, driven by Brownian motion, and the spheres returned to a dispersed state.

At sphere volume fractions $\approx 25 \%$ the structure formation was investigated under two different starting conditions. In one case, the particles were allowed to sediment under gravity (figure 8a) in the absence of a field. The bottom layers of the sediment were observed to be randomly stacked hexagonal close-packed planes [18,19, and the upper layers of spheres were in a fluid-like state. When an electric field $(\approx 1 \mathrm{kV} / \mathrm{mm}, 500 \mathrm{kHz})$ was applied across the electrode gap of $\approx 70 \mu \mathrm{m}$, the spheres at the interface of the sediment and solvent began to form field aligned chains that eventually reached the upper electrode. This occurred within a few (2-3) minutes. Over the next several hours the spheres in the entire sediment 
rearranged into field aligned chains that attracted each other to form columns that spanned the electrodes (figure 8b). The sphere arrangement within the columns was a BCT structure. The columns themselves were bridged together by domains of BCT crystals. After coarsening for a few hours further development of the column-structures stopped and no further evolution occurred over a period of 2 days. Lowering the electric field slowed down the structure formation. We did not see sheet-like structures under these initial conditions.

When the electric field was applied across a solution with volume fraction $\approx 30 \%$, but with the spheres initially dispersed through the solvent, we observed a pattern of structure formation similar to that seen at lower concentrations with similar starting configurations, where labyrinths of small sheet-like structures, along with isolated chains of spheres developed within seconds. The sheets evolved into a collection of small sections of interconnected BCT structures that retained the labyrinthine appearance of the sheets. When the field was turned off, the structures disappeared within seconds, as was the case in all our observations.

Observations were made at higher concentrations, volume fraction $\approx 45 \%$ where the spheres crystallize. In the absence of electric fields the spheres were arranged in FCC stacked hexagonal layers parallel to the electrodes, with the top few layers being liquid-like [18,19]. As is the case in our observations, it has been observed in other experiments that, except for the hard-sphere limiting case (thin steric stabilizer layer or very thin double layer with 0.1 M salt), the crystal structure formed upon sedimentation is FCC [20]. When an electric field $\approx 1 \mathrm{kV} / \mathrm{mm}$ and $500 \mathrm{kHz}$ was applied across the electrode gap $(\approx 80 \mu \mathrm{m})$, defects appeared in the hexagonal structure, and areas of BCT formed in the bulk of the sample over the first $\approx 10$ minutes. After a few hours it was observed that sections of the colloidal crystal had transformed into BCT order in the bulk of the sample, with the bottom 5-6 layers remaining hexagonal, and the top 2-3 layers remaining disordered. There were no sheets observed at this concentration. Figure 9 shows the transition from hexagonal ordering in the absence of a field, to a mix of BCT and hexagonal order after the field was on for $\approx 6$ hours. It should be noted that, as indicated in the upper portion of Fig.9b that there is free space between BCT crystals of different orientation. When the field was switched off the BCT 
crystals stayed in the same symmetry but expanded to become 100 oriented FCC crystals, as opposed to the initial 111 FCC symmetry seen before the field was turned on. When the field is switched on again the crystal goes through a martensitic transition back to BCT. This ability to tune the crystal structure by using an electric field could have applications in the field of photonic crystals.

\section{DISCUSSION}

It is interesting to consider other studies that address the issue of structure formation. Labyrinthine structures similar to sheets have been observed in ferrofluids, but the structure within the sheets was not ascertained [21]. The presence of hexagonal sheets has not been reported in previous experimental observations on ER fluids, but has been observed in simulations [11, 12, 22, 24]. The simulations of Martin, Anderson and Tigges [11, 12] show that for sphere concentrations less than $\approx 30 \%$ a sudden application of the field first induces formation of short chains parallel to the field. The chains then attract each other, forming sheets, which often are bent into tube and spiral-like forms, or form thick walls . This was in contrast to our observations, where the sheets remained two-dimensional for long periods of time. Very few BCT domains were found in the simulations that neglected thermal motion [11], probably because the sheets were prevented from annealing into the ground state BCT crystal. This is in contrast to our experiments, where large BCT crystals formed after long exposures to the field. In their simulations that considered thermal effects [12], Martin, Anderson and Tigges observed increased order, crystallinity, and larger domain sizes compared to their simulations neglecting thermal motion [11]. However a sheet to BCT transition was not seen, possibly because of the insufficient duration of the simulation. The structure evolution we observed followed the general pattern of the simulations [11,12], although we did not observe the sheets to form from the association of chains. However, the time resolution of our observations was not sufficient to study the initial association in more detail.

Halsey and Toor [10,25] studied structure evolution by considering that the shape of 
a particle aggregate would be a droplet, modeled as a prolate spheroid. The droplet is assumed to grow with time, in a quasi-equilibrium manner, as individual particles attach to it. It elongates towards the electrodes as it grows, with its shape being determined by balancing the bulk and surface electrostatic energies due to the dipoles it contains, forming into a column spanning the electrodes. Individual columns will aggregate over time towards a bulk phase segregation. This model assumes the concentration of particles is low, and that the droplet is always in equilibrium, which requires that the electric field is low compared to thermal energies. They point out that the equilibrium droplet model fails at high fields, where columns will form rapidly in a non-equilibrium manner but growth will become arrested before equilibrium bulk phase separation occurs. We did not observe droplet-like structures. At the volume fractions we used, and with the rapidly quenched relatively high electric fields we used, structure formation did not occur particle by particle in a quasi-equilibrium manner as in the droplet model, but rather as a association of chains and sheets. We observed column formation at volume fractions of $\approx 25 \%$ and with the colloid initially sedimented, but the columns did not coarsen to allow bulk phase separation over a period of observation of 2 days. Martin and colleagues have performed two-dimensional light scattering studies on a model ER fluid using particles and field strengths similar to those used by us [26]. They observed a two-stage, chain to column structure formation under conditions of no shear. Although sheet formation might be expected under the conditions used, they did not observe sheets. This may be because light scattering methods alone are insufficient to detect and interpret sheet-like structures.

Melrose [22,23] has performed Brownian dynamics simulations on ER fluids of particle volume fractions ranging from $\approx 10 \%$ to $50 \%$. For the case where no shear was applied on the ER fluid, particles were observed to form into strings, which subsequently aggregated together. At $10 \%$ volume fraction, strings and small aggregates of strings are seen. At $30 \%$ v.f. a kinetically arrested gel is seen, where the gel is composed of hexagonal sheets of particles. The hexagonal arrangement within the sheets is not described. The sheets form into a labyrinthine structure similar to that seen in our observations. The simulation remains 
trapped in a local potential minimum, and is not seen to evolve further towards a crystalline ground state. In this simulation, Brownian motion was turned off upon application of the

electric field. A similar kinetically trapped gel-like state was observed in the simulations of Hass [24], who did not observe the ER fluid to evolve into a regular lattice. Brownian effects were neglected in this simulation.

A Brownian dynamics simulation by Tao and Jiang [27] on a system consisting of 122 particles (with volume fraction $\geq 20 \%$ ), which considers thermal motion during structure formation, shows the ER fluid rapidly forming chains, which aggregate into thick columns. These columns consist of polycrystalline BCT lattice grains that are aligned along the field direction, but misaligned in the xy direction. We observed similar column-like structures at $\approx 25 \%$ volume fraction. When thermal forces are neglected, the ER fluid is described to be trapped in a local minimum energy state. However, a sheet-like state is not reported as an intermediate state during structure development with thermal forces included.

\section{CONCLUSION}

We have verified through direct visualization that our model ER fluid reaches the BCT structure as a ground state under most conditions, and have been able to describe structure formation as a function of concentration. At the lowest concentrations observed (about 10\%), BCT crystals were seen primarily to form through chains of particles attracting each other, while a smaller fraction of particles formed sheets that transformed into BCT structures by annealing together. As the concentration was increased beyond 15\% the presence of chains decreased and sheets dominated at early times, forming into complex labyrinthine structures which lasted for hours before annealing into BCT crystals. At high concentrations (larger than 40\%), where the initial structure was FCC, BCT crystals were formed via defects appearing in the existing hexagonal structures. The unusual symmetry of the BCT crystals and the martensitic FCC-BCT crystal transition both promise applications in photonics. Unlike magnetorheological fluids, ER fluids can be made from non light-absorbing materials. The martensitic crystal switching we observed was on structures of size of the order of the 
wavelength of light, as opposed to the FCC-BCT transition of $\approx 45 \mu \mathrm{m}$ sized spheres described recently [3]. The column-like structures observed at intermediate concentrations $(\approx 25 \%)$, were interlinked in complex ways through strings of particles and BCT crystals, and had wide variation in size. The structure within the columns was that of domains of BCT which were misaligned in the plane normal to the electric field. We observed that at a low concentration there was a sample thickness (about 10 sphere diameters) below which the preferred state was that of strings and sheets, and BCT crystals did not form.

Data and analyzed images of our observations are available on the world wide web at http://www.elsie.brandeis.edu.

Acknowledgment: This work was supported by the United States Department of Energy under Grant No. DE-FG02-94ER45522 and NSF International Travel Grant INT-9113312 and is part of the research program of the "stichting voor Fundamenteel Onderzoek der Materie (FOM)", which is financially supported by the "Nederlandse Organisatie voor Wetenschappelijk Onderzoek (NWO)". 


\section{REFERENCES}

[1] J. E. Stangroom, Phys. Technol. 14, 290 (1983)

[2] A. van Blaaderen, MRS Bulletin 23(10), 39 (1998)

[3] W. Wen, N. Wang, H. Ma, Z. Lin, W. Tam, C. Chan and P. Sheng Phys. Rev. Lett. 82, 4248, (1999)

[4] J. Priestley, The History and Present State of Electricity with Original Experiments 2nd ed. (London, 1769)

[5] H. A. Pohl, Dielectrophoresis p.495, (Cambridge University Press, 1978)

[6] W. M. Winslow, J. Appl. Phys. 20, 1137 (1949)

[7] M. Parthasarathy and D. Klingenberg, Materials Science and Engineering R17, 57 (1996)

[8] R. Tao and J. M. Sun, Phys. Rev. Lett. 67, 398 (1991)

[9] T. Chen, R. N. Zitter and R. Tao, Phys. Rev. Lett. 68, 2555 (1992)

[10] T. C. Halsey and W. Toor, Phys. Rev. Lett. 65, 2820 (1990)

[11] J. E. Martin, R. A. Anderson and C. P. Tigges, J. Chem. Phys. 108, 3765 (1998)

[12] J. E. Martin, R. A. Anderson and C. P. Tigges, J. Chem. Phys. 110, 4854 (1999)

[13] A. van Blaaderen, R. Ruel, and P. Wiltzius, Nature 385, 321 (1997)

[14] A. van Blaaderen and P. Wiltzius, Science 270, 1177 (1995)

[15] A. van Blaaderen and A. Vrij, Langmuir 8, 2921 (1992)

[16] T. Wilson(ed.), Confocal Microscopy, (Academic Press, London, 1995)

[17] J. M. Sun and R. Tao, Phys. Rev. E 53, 3732 (1996) 
[18] P. N. Pusey, W. van Megan, P. Bartlett, B. J. Ackerson, J. G. Rarity and S. M. Underwood, Phys. Rev. Lett. 63, 2753 (1989)

[19] M. S. Elliot, B. T. F. Bristol and W. C. K. Poon, Physica A 235, 216 (1997)

[20] A. van Blaaderen, unpublished results

[21] E. M. Lawrence, M. L. Ivey, G. A. Flores, J. Liu, J. Bibette and J. Richard, International Journal of Modern Physics B 8, 2765 (1994)

[22] J. R. Melrose and D. M. Heyes, J. Chem. Phys. 98, 5873 (1993)

[23] J. R. Melrose, Mol. Phys. 76, 635, (1992)

[24] K. C. Hass, Phys. Rev. E 47, 3362, (1993)

[25] W. Toor, Journal of Colloid and Interface Science 156, 3351993

[26] J. E. Martin, J. Odinek, T. C. Halsey and R. Kamien Phys. Rev. E 57(1), 756, (1998)

[27] R. Tao and Q. Jiang, Phys. Rev. Lett. 73, 205, (1994) 
FIGURES

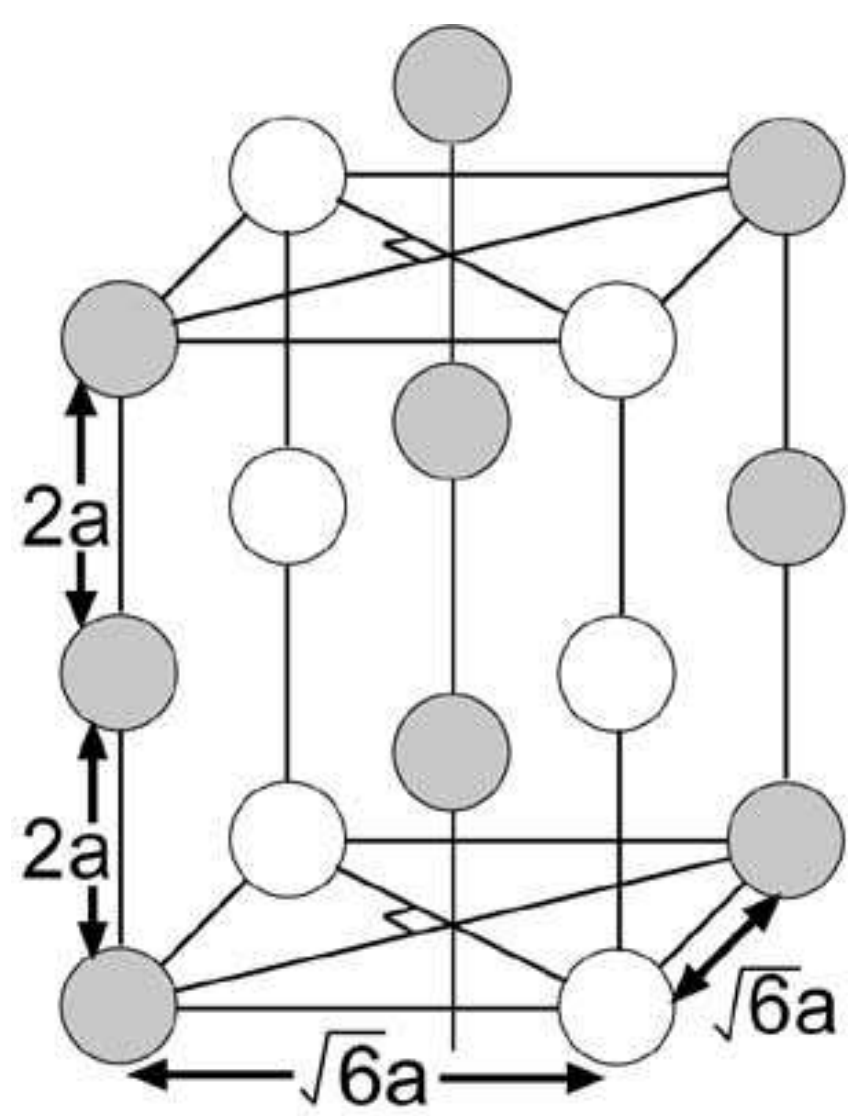




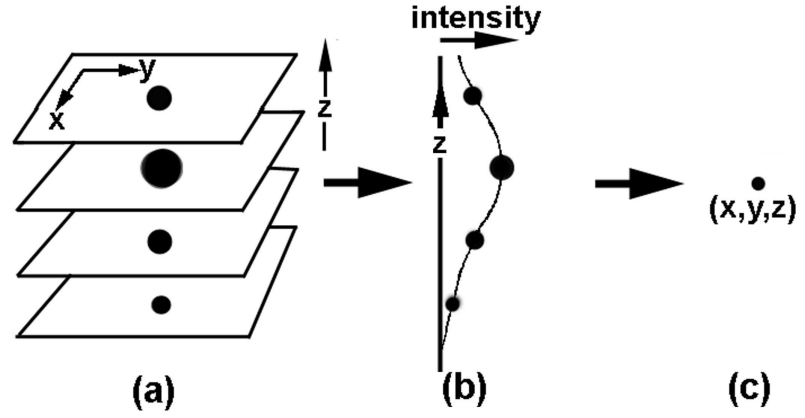




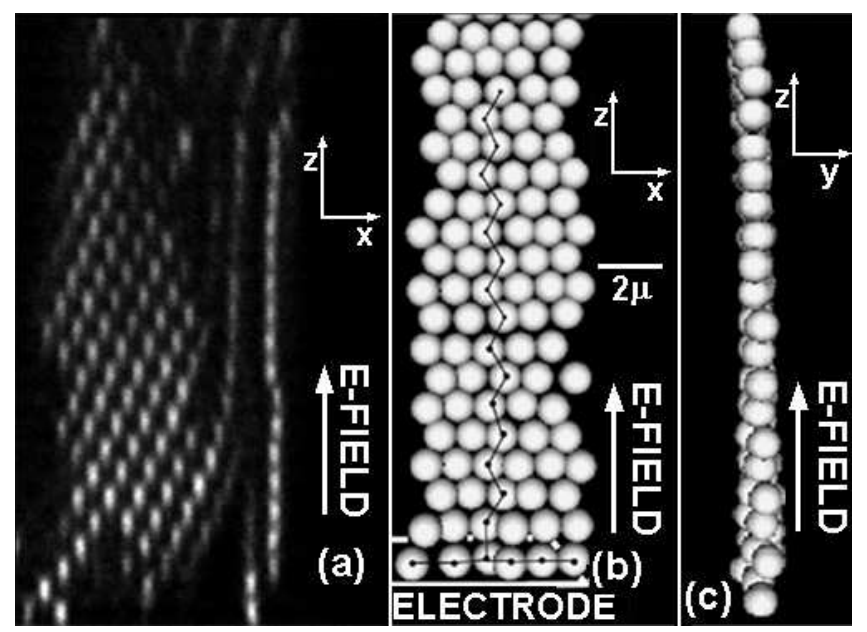




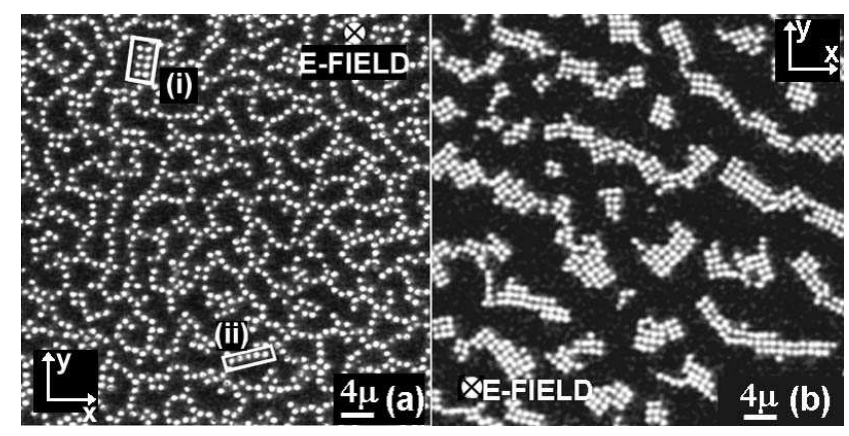




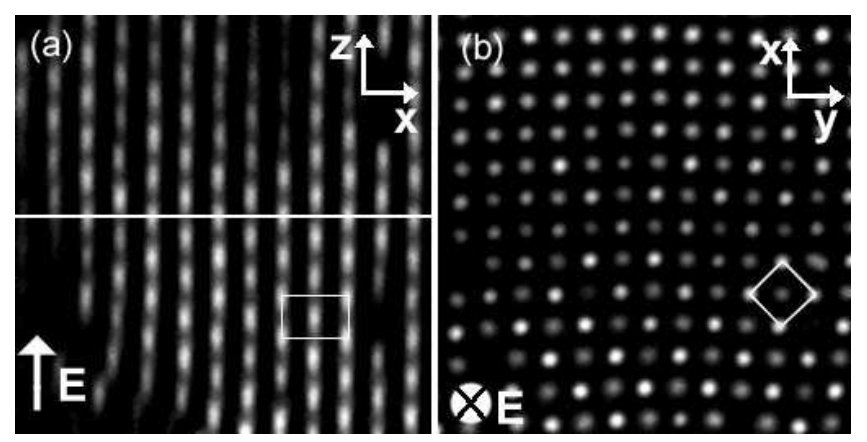




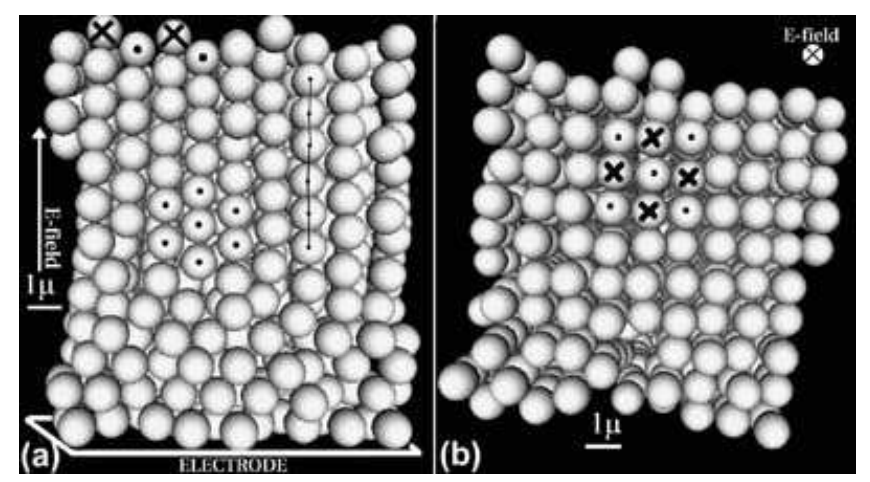


TABLES

\begin{tabular}{lc}
\hline \hline Structure & Energy per dipole \\
\hline Separated chains & -0.3005 \\
Cubic lattice & -0.2618 \\
Sheet & -0.3448 \\
BCC & -0.3401 \\
FCC & -0.3702 \\
HCP & -0.3707 \\
BCT & -0.3813 \\
\hline \hline
\end{tabular}




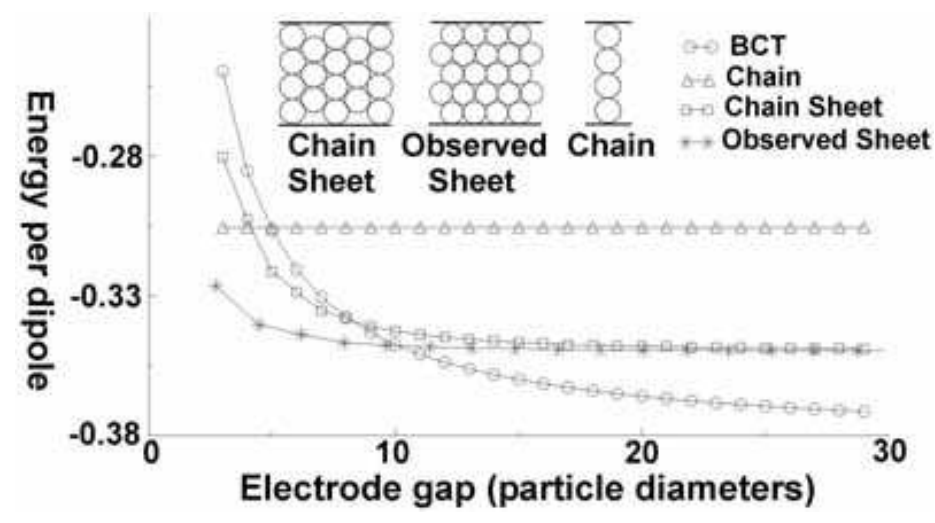




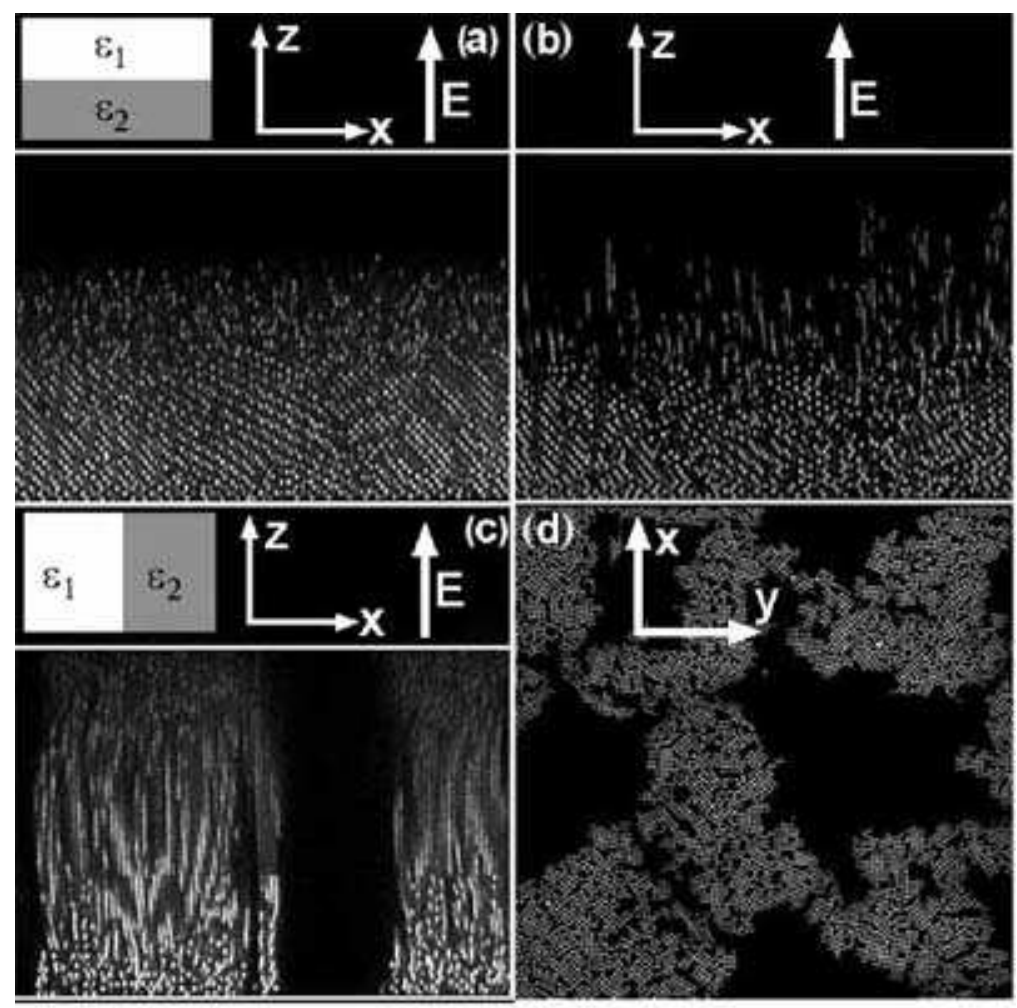




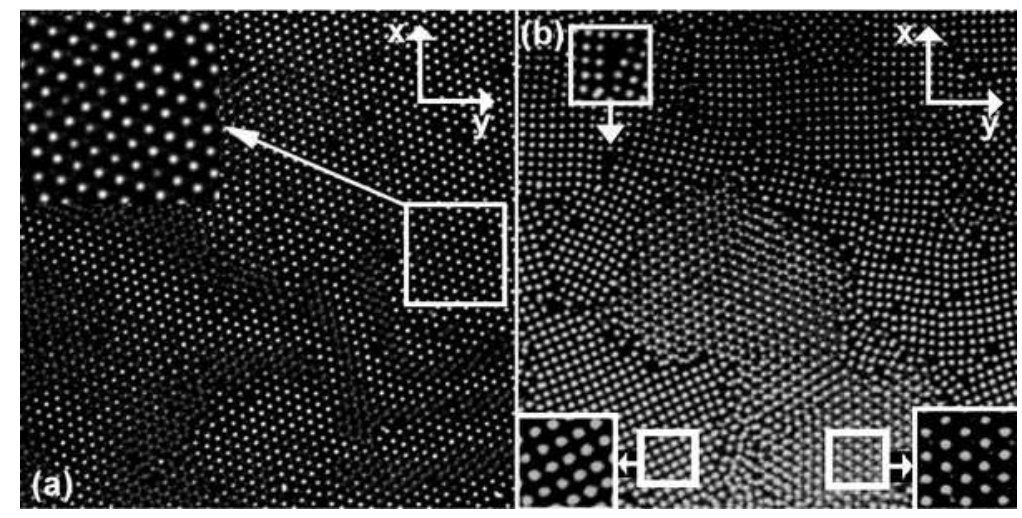


FIG. 1. The three-dimensional BCT structure. The spheres have radius $a$ (the spheres are not drawn to scale). The crystal can be regarded as a collection of close-packed planes. One such plane is indicated by the shaded spheres. Each close-packed plane is a collection of chains of touching spheres, where neighbouring chains are offset from each other by a particle radius. In the ground state of ER fluids the chains are aligned with the electric field, thus a section perpendicular to the electric field shows a square arrangement of spheres (see fig.5b). The volume fraction of a BCT crystal is 0.698 .

FIG. 2. Particle finding algorithm. A diagram showing the method of obtaining particle coordinates from the confocal micrographs. The $\mathrm{z}$ axis is along the optical axis and the electric field. (a)Each particle gives rise to roughly circular regions of varying intensity in neighbouring image planes which are separated by $\approx 0.1 \mu \mathrm{m}$. The xy centroid coordinate of each region is obtained by averaging coordinates weighted by intensity. (b)The regions belonging to an individual particle are identified. (c) The final xyz coordinate is found by an intensity-weighted average along each axis of the regions identified in (b).

FIG. 3. Sheet structure. The field strength is $1.2 \mathrm{kV} / \mathrm{mm}$ and the field has been on for $\approx 30$ minutes. (a) $\mathrm{x}-\mathrm{z}$ Confocal micrograph (raw data). There is a sheet of particles seen in the left side of the image, with a single chain of particles to the right of it. The bottom of the image is closest to the objective lens of the microscope. It should be noted that since only the cores of the silica spheres fluoresce, touching spheres are seen as separated. It is the anisotropic point spread function that causes the spherical fluorescent cores to appear ellipsoidal. Note also that the intensity and resolution diminishes away from the lens due to spherical abberation.(b) View of a sheet seen face-on. The image is taken from a rendering of reconstructed 3-d raw data such as seen in (a). The white scale bar is $2 \mu \mathrm{m}$. The bottom row of spheres is touching the glass electrode (the electrode is perpendicular to the image), and the electric field is upwards. The spheres are hexagonally close packed within a sheet. The smallest angle between nearest neighbours and the field is $\pm 30^{\circ}$, except for the first two layers adjacent to the electrode, where nearest neighbours are aligned along the field. (c) Side view of the same sheet object. 
FIG. 4. Labyrinth of sheets (a)View of a labyrinth of sheets looking down the electric field. The images are raw $\mathrm{x}-\mathrm{y}$ confocal micrographs. The point spread function is symmetric in the plane $(\mathrm{xy})$ perpendicular the objective. The view is that seen after $\approx 3$ minutes of the sample (volume fraction 15\%) being in the E-field. The sheets form within seconds of the E-field being turned on, and evolve into the BCT structure over hours, indicated by (i) in the image above. The structure of a sheet such as indicated by (ii) in the image is shown in figures $3 \mathrm{~b}$ and $3 \mathrm{c}$. The white bar is $4 \mu \mathrm{m}$. Note that the raw data only shows the (fluorescent) cores of the spheres, making it possible to distinguish touching spheres. Spheres within $\pm 0.5 \mu \mathrm{m}$ of the image plane contribute to the image. The image is from a plane $20 \mu \mathrm{m}$ from an electrode in a sample with an electrode gap of $70 \mu \mathrm{m}$. (b) After several hours, the sheets such as seen in (a) anneal together and form collections of BCT structures. The field is perpendicular to the image. Each BCT cluster extends long distances in the field direction.

FIG. 5. Body Centered Tetragonal crystal. Raw 3D data set of a BCT crystal consisting of $13 \mu \mathrm{m} \times 13 \mu \mathrm{m} x-y$ planes separated in the z-direction by $0.09 \mu \mathrm{m}$. The image (a) is a digital interpolation of data from a sequence of $x-y$ planes such as seen in (b). (a) shows a view along a plane parallel to the E-field, showing the centered rectangular lattice of dimension $2 \sqrt{3} a \times 2 a$ (110 plane) of the BCT lattice, where the spheres have radius $a$. In this plane the BCT structure consists of chains of spheres aligned along the field direction. Neighbouring chains are offset along the field by one particle radius. (b) shows a view looking down the E-field showing the square $\sqrt{6} a \times \sqrt{6} a$ lattice (001 plane) of the BCT lattice. The plane (b) is a section orthogonal to (a) such as along the line indicated in (a). The alternating intensity pattern seen in (b) arises because adjacent chains of spheres are offset in and out of the plane by one particle radius.

FIG. 6. BCT Crystal. Two views of a BCT crystal rendered in 3D after obtaining coordinates of the centers of each particle from raw data such as seen in Figure 5. The view is of a portion of a larger crystalline region. (a) shows a view looking in a plane parallel to the E-field. The particles connected with a line show a chain parallel to the E-field. 
The view shows the hexagonal 110 plane of the BCT crystal. As in (b), the $\mathrm{x}$ and $\bullet$ show neighbouring chains of particles that are out of register by a particle radius. The spheres in the first few layers adjacent to the electrode are arranged in random stacked hexagonal planes parallel to the electrode. (b) shows a view perpendicular to the E-field. The $\bullet$ and $\mathrm{x}$ symbols represent chains of particles aligned with the E-field, and chains marked with a • are out of register with the $\mathrm{x}$ chains by one particle radius.

Table 1: Dipolar energy per particle for various infinite sized lattices. Energy is in units of $p^{2} / a^{3} \epsilon_{f}$, where the dipole moment $p=a^{3} \epsilon_{f} E\left(\epsilon_{p}-\epsilon_{f}\right) /\left(\epsilon_{p}+2 \epsilon_{f}\right) . \epsilon_{p}$ and $\epsilon_{f}$ are the dielectric constants of the particle and solvent respectively, and $\mathrm{E}$ is the magnitude of the electric field.

FIG. 7. Dipole energy per particle vs. electrode gap. Image charges are included in this calculation. The energy per particle is in units of $p^{2} / a^{3} \epsilon_{f}$, where $z p=a^{3} \epsilon_{f} \mathrm{E}\left(\epsilon_{p}-\epsilon_{f}\right) /\left(\epsilon_{p}+2 \epsilon_{f}\right)$. $\epsilon_{p}$ and $\epsilon_{f}$ are the dielectric constants of the particle and solvent respectively, and $\mathrm{E}$ is the magnitude of the electric field. 'Observed sheets' are sheets with the configuration observed in the experiments, while 'chain sheets' are close-packed planar sheets consisting of chains of spheres aligned along the electric field, where neighbouring chains are offset along the electric field direction by one particle radius. At an electrode spacings of less than 10 particle diameters the observed sheets have a lower energy than the BCT crystal.

FIG. 8. Field response of sedimented colloid. (a) At a particle volume fraction of $\approx 25 \%$ the colloid is left to sediment to the bottom electrode before a E-field is applied. The horizontal white lines represent the transparent electrodes. (b) Shows the sedimented sample as shown in (a) transforming into a columnlike structure upon application of a Efield. The image is taken 2 minutes after the field was on. Chains of particles are seen to form along the field. (c) After a few hours the spheres arrange into cross-linked columns formed parallel to the field. (d) A view of the columns from a plane perpendicular to the field. The square-like arrangement of the particles within the columns indicates that the structure within the columns is BCT. If the sphere-rich region and solvent are treated as dielectric fluids with different dielectric constants placed between the plates of a parallel plate capacitor, then the configuration seen in (c) (and shown schematically in the upper 
left corner of $(\mathrm{c})$ ), where the fluids are separated into regions with their interfaces parallel to the field, has lower electrostatic energy than the configuration in (a), where the fluids are separated in the sedimented state. This explains the tendency to form columns.

FIG. 9. Field induced solid-solid transition. The image shows raw confocal microscope data of a sample of volume fraction $\approx 45 \%$. (a) Shows a plane parallel to the electrodes before the E-field was turned on. The spheres are arranged as hexagonal planes with many defects, parallel to the electrodes. The structure is FCC. The image is from a plane $20 \mu \mathrm{m}$ from an electrode, and the electrode gap is $80 \mu \mathrm{m}$. (b) Shows the same area about 6 hours after an E-field is applied (perpendicular to the image plane). Large areas of the crystal have transformed into BCT order, identified by the square configurations. 